\title{
Puffer fish poisoning: a case report
}

\author{
Khan $\mathrm{MI}^{1}$, Habib $\mathrm{R}^{2}$, Islam $\mathrm{MD}^{3}$, Newaz $\mathrm{MS}^{4}$
}

\begin{abstract}
Puffer fish poisoning after consumption of this fish is one of the most common causes of poisoning among people in the coastal regions of Asia. Puffer fish poison (tetradotoxin) is one of the most toxic substances still found on earth; it is about 275 times more lethal poison than cyanide and fifty times more potent than strychnine or curare poison. Still no anti toxin or antidote is being discovered yet. On 6th January 2021, among five members of a single family, all of them ingested puffer fish after cooking, everyone developed toxic manifestations. Father died after three hours on the way to hospital, mother died after 17 hours during treatment and three daughters improved without any residual complication after getting supportive treatment in this hospital.
\end{abstract}

CBMJ 2019 January: vol. 08 no. 01 P: 35-38

Key words: Puffer fish poisoning, Tetradotoxin.

\section{Introduction}

The puffer fish is the most common fish poisoning along the coastal region of the Asia Pacific and has been reported from ancient times. ${ }^{1,2}$ Puffer fish poisoning has been documented in many Asian countries including Thailand, Malaysia, Bangladesh, Taiwan and particularly Japan. ${ }^{3}$ Puffer fish, also known as blow fish, balloon fish, toad fish, globe fish, fugu etc. among the different parts of the world. ${ }^{4}$ Puffer fish gets its name from its inflatable nature wherein it can swallow a large amount of water when facing any kind of danger thereby resembling a huge ball which is far bigger than the fish's original size. ${ }^{5}$ All species belong to the order tetrodontiformes. ${ }^{6}$ In Bangladesh, it is popularly named as potka fish as it can replicate a huge balloon. Because of its low cost and availability, and also a delicious dish, potka fish is popular, particularly among the people in the poor rural communities.

The toxin of the puffer fish poisoning is identified as tetrodotoxin (TTX) and poisoning is caused by sufficient amount ingestion of the liver, gall bladder, intestine, gonads, skin of the fish to cause intoxication. ${ }^{8}$ The flesh of this fish is less poisonous. ${ }^{9}$ All species of puffer fish are not toxic, and some of them are only mildly toxic. $^{10}$ The severity of toxicity may vary with sex, season and geographical location. During reproductive period the ovaries of the female fish are more toxic than the male testes, and the toxicological pattern also varies between temperate and tropical zone. ${ }^{10}$ The world famous Captain Cook was poisoned by it's ingestion in 1774 and the voodoo poisons of Haitian folklore responsible for zombification reportedly contains the poison "tetrodotoxin". ${ }^{11,12}$ Tetrodotoxin is also found in the gastropod mollusc; eggs of horseshoe crabs; newts of the genus Taricha; skin of Atelopid frogs;

1. Dr. Mohammad Monirul Islam Khan, Assistant Professor (Pediatrics), Shaheed Syed Nazrul Islam Medical College, Kishoreganj

2. Dr. Rahat Bin Habib, Assistant Professor (Pediatrics) Shaheed Syed Nazrul Islam Medical College, Kishoreganj

3. Dr. Mohammad Didarul Islam, Assistant Professor (Pediatrics) Shaheed Syed Nazrul Islam Medical College, Kishoreganj

4. Dr. Md. Shah Newaz, Registrar (Pediatrics) Shaheed Syed Nazrul Islam Medical College Hospital, Kishoreganj

Address of correspondence:

Email:mikhan29@gmail.com

Mobile: +8801711361340 
skin and viscera of porcupine fish, globefish, balloon fish, blowfish, sunfish, toadfish, blue-ringed octopus, and some species of salamanders. ${ }^{13}$ The toxin is also used as a defensive biotoxin to ward off predation, or as both defensive and predatory venom. Japanese scientist Dr. Yoshizumi Tahara first isolated and named the toxin tetrodotoxin in $1909 .^{13}$

Several reports are published sporadically for the episodes of puffer fish toxicity in Bangladesh, primarily from districts in the coastal region. In Bangladesh, till date 13 species of the fish are isolated; among them two live in fresh water whereas the others are marine puffer fish. ${ }^{14}$ Although there are reports on toxicological analysis of two fresh and three marine water species of puffer fish, information about the other species of puffer fish in Bangladesh is not available. ${ }^{15}$

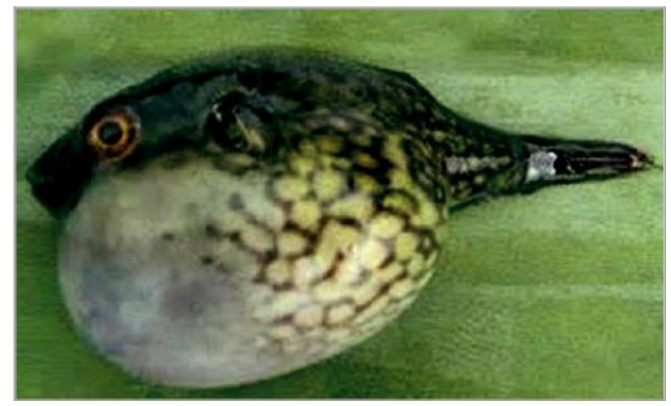

Figure 1: Puffer fish after ingestion of huge water resembling ball.

\section{Case summary}

A 9 years old female child admitted in Shaheed Syed Nazrul Islam Medical College, Kishoreganj with the complaints of tingling and perioral numbness which then spread all over the body, itching of the mouth, vomiting for several times containing undigested food materials, and restlessness about five hours after ingestion of puffer fish, and also respiratory distress for about one hour. Her father died after three hours of ingestion of puffer fish while on the way to hospital and mother died after 17 hours of ingestion while getting treatment in this hospital. Her elder sister aged 18years was also admitted in the same hospital with same diagnosis and receiving treatment. After admission, she was slightly drowsy and restless, examination revealed pulse 60 beats $/ \mathrm{min}$, blood pressure $90 / 60 \mathrm{~mm}$ of $\mathrm{Hg}, \mathrm{SpO}_{2} 97 \%$ in room air, temperature normal, pupils were normal in size and reacting to light. She was not cyanosed, not dehydrated, higher psychic functions were intact except slightly drowsy and anxious, her cranial nerves were intact, motor, sensory functions and reflexes were within normal limit.

She was treated with symptomatic and supportive treatment with nothing per oral, 6-10 liter/minute oxygen inhalation by face mask, nutrition maintained by intravenous fluid, injection omeprazole, neostigmine, atropine, hydrocortisone, and ceftriaxone. Due to delayed presentation, no gastric lavage was given. Within 24 hours her clinical condition improved, no respiratory distress, no nausea-vomiting, no tingling sensations. Then oral feeding was started with liquid and semisolid diet, oxygen no longer required. Her baseline investigations (CBC, LFT, Serum electrolyte, serum creatinine) were within normal limit. She and her two elder sister (aged 13 years and 18 years) got improved without any residual complication and were discharged from hospital 5th day after admission.

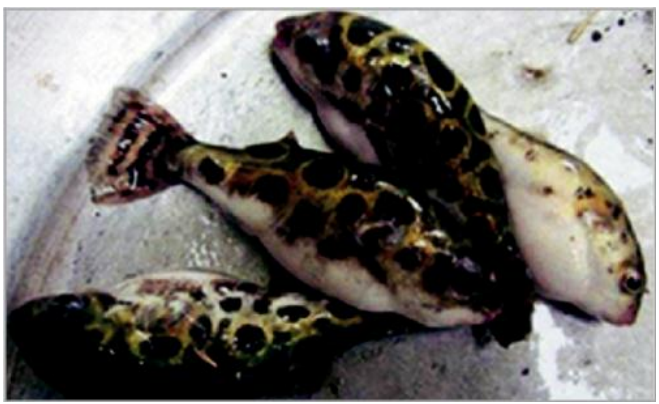

Figure 2: Puffer fish species found in Bangladesh. 


\section{Discussion}

Cases of puffer fish poisoning may not be unusual in our country as there is no rules and regulations or supervision on puffer fish culture, sales and cooking, where as in Japan it is highly regulated.

A person may develop the signs and symptoms of puffer fish poisoning within 30 minutes to five hours after consuming the fish. The part of the fish and the amount of tetrodotoxin present in the fish consumed determines how rapidly the symptoms occur and its severity. ${ }^{5}$

Tetradotoxin is heat stable, water soluble and a non-protein quinazoline derivative type of neurotoxin. ${ }^{6}$ It blocks the sodium pump and neuronal transmission in the all excitable cell membrane, and thus all the toxicity is secondary to blockage of development of action potential ${ }^{1}$ leading to a tingling sensation in the lips and tongue followed by perioral numbness, soon after the whole face, even in the extremities start feeling numbness, feeling of intoxication and dizziness, slurring of speech, limbs start getting paralyzed, respiratory distress, heart rate and respiratory rate become abnormal and there may be lowering of blood pressure. ${ }^{5}$ It also stimulates the chemoreceptor trigger zone (CTZ) in the medulla oblongata, causing pain in the abdomen, nausea, vomiting, diarrhea and other symptoms related to the gastrointestinal tract disturbances start to occur rapidly, and depress the respiratory and vasomotor centers also. ${ }^{6}$ Severe toxicity can lead to convulsions and the person might become unconsciousness or even go into deep coma. ${ }^{5}$ Death usually results from respiratory failure because of paralysis of respiratory muscles. ${ }^{4}$

No specific laboratory test that confirms puffer fish ingestion and tetrodotoxin poisoning exists; thus, dietary history is the key for confirmatory diagnosis. Tetrodotoxin may be detected by fluorescent spectrometry but still it is not available in Bangladesh. ${ }^{13}$
Till date, tetrodotoxin poisoning has no specific antidote or antitoxin, ${ }^{6}$ only immediate supportive treatment, that is early intubation, circulatory support and judicious administration of anticholinesterase drugs (neostigmine), along with atropine, can minimize its paralyzing effects. $^{16}$

\section{Conclusion}

In Bangladesh, because of its low cost and availability and is also an extremely delicious dish, there is every chance of puffer (potka) fish poisoning. The cooking process of puffer fish requires a special technique, of which most people are unaware about it. As it has no specific treatment, every individual should be very much cautious before eating the delicious puffer fish and should be made aware of the potential toxic effects. Efforts to prevent future puffer fish intoxication should include wider dissemination of public health messages using audio-visual mass media such as radio, television, miking in fish market, speech by local imam in mosque, local political leader, electronic and print media, and staging folk songs, palagaan, drama etc. Local public health administration should take necessary action to improve awareness among fish sellers and fish distributors not to culture, catch, sell, buy any puffer fish and also give training health personnel to face this sort of medical emergency as early diagnosis and immediate supportive management could ensure a safe and favorable outcome.

\section{References:}

1. Mills AR, Passmore R. Pelagic paralysis.Lancet. 1988; 1:161-164.

2. Chew SK, Goh $\mathrm{CH}$, Wang $\mathrm{KW}$ et al. Puffer fish (Tetrodotoxin) poisoning: clinical report and role of anticholinesterase drugs in therapy. Stand Med J. 1983; 24:168-171.

3. Tsunenari $S$, Uchimura $Y$, Kanda $M$. Puffer poisoning in Japan - a case report. J ForensicSci. 1980; 25: 240-245.

4. Benzer T. Tetrodotoxin. Engl Med J. 2001;2:1-9. 
5. Kerkar $P$. In puffer fish poisoning. Symptoms, causes, treatments. 2017. [available from: https://www.epainassist. com/stings-and-bites/puffer-fish-poisoning].

6. Sun Diego Department of environmental health, FDA. Tetrodotoxin poisoning associated with eating puffer fish transported from Japan to California, 1996. Morb Mortal Wkly Rep. 1996; 45:389-91.

7. Chowdhury FR, Mamun AA. A fishy tale. Student BMJ. 2004; 12:349-92.

8. Chowdhury FR, Ahsan HA, Mamun AA, Rashid AK, MahboobAA. Puffer fish (Tetrodotoxin) poisoning: an analysis and outcome of six cases. Trop Doct. 2007;37:263-264.

9. Ahsan HA, Mamun AA, Karim SR, Bakar MA, Gazi EA, Bala CS. Paralytic complications of puffer fish (tetrodotoxin) poisoning. Singapore Med J. 2004; 45:7374.

10. Lee MJ, Jeong DY, Kim WS, Kim CS, Kim $H D$, Park WW, Kim KS, Kim DS. A tetrodotoxin producing vibrio strain, $L M-1$, from the puffer fish fugu vermicularis radiatus. Appl Environ Microbiol. 2000;66:1698-1701.

11. Beaglehole JC. The journals of Captain James Cook on his voyages of discovery. Vol.2. The voyages of Resolution and Adventure, 1772-1775. Cambridge:Cambridge University Press. 1961:534-5.

12. Davis EW. The ethnobiology of the Haitian zombi. J Ethnopharmacol. 1983; 9:85-104.

13. Hoque MA, Islam TQ, Ekram SANM. Puffer fish poisoning. TAJ. 2008; 20(2): 199-202.

14. Diener M, Christian B, Ahmed MS, Luckas B. Determination of tetrodotoxin and its analogues in the puffer fish Takifugu Oblongus from Bangladesh by hydrophilic interaction chromatography and mass spectrometric detection. Anal Bioanal Chem. 2007; 389: 1997-2002.

15. Mahmud $Y$, Tanu MB, Noguchi T. First occurrence of a food poisoning incident due to ingestion of Takifugu oblongus, along with a toxicological report on three marine puffer species in Bangladesh. Journal of the food Hygienic society of Japan.1999; 40: 473 480.
16. Chew SK, Chew LS, Wang KW, Mah PK, Tan BY. Anticholinesterase drugs in the treatment of tetradotoxin poisoning. Lancet. 1984; 2:109. 